\title{
Ueber die mittlere Parallaxe der Sterne erster Grösse.
}

Im Anschluss an den $>$ Versuch einer Ableitung der Bewegung des Sonnensystems aus den Potsdamer spectrographischen Beobachtungen e erlaube ich mir im Folgenden das Ergebniss der Verwerthung derselben Beobachtungen zur Bestimmung der mittleren Parallaxe der Sterne erster Grösse ganz kurz zu erwähnen. Zu Grunde gelegt ist der Satz von J. Kleiber, dass man die Summe der Bewegungen in Rectascension gleich der in Declination und gleich der Summe der Bewegungen in der Gesichtslinie setzen kann. Jene scheinbaren Bewegungen müssen erst durch die Quadratwurzel der Helligkeit dividirt werden, um für alle Sterne die gleiche Grössenklasse zu erhalten. Ich habe die photometrischen Messungen von Dr. Th. Wolff hierbei benutzt.

Berlin, Kgl. Recheninstitut, 1893 Febr. 9.
Eine grosse Genauigkeit bei dem Resultat ist natürlich nicht zu erwarten, ich habe sie daher auch nicht erstrebt und ftihre nur an,

$$
\begin{array}{ll}
\text { dass die Rectascensionen } & \pi=0.080 \\
\text { und die Declinationen } & \pi=0.059
\end{array}
$$

also im Mittel die Parallaxe eines Sterns erster Grösse (von der Helligkeit von $\alpha$ Lyrae) gleich 0.07 ergeben haben, nahezu mit J. Kleiber's Werth in A. N. 3037 ubereinstimmend. Obschon ziemlich klein, widerspricht diese Zahl keineswegs den directen Parallaxenbestimmungen, die sich vorwiegend auf Sterne mit grosser Eigenbewegung und daher relativ grosser Parallaxe bezogen haben.

\section{Elemente und Ephemeride des Planeten (241) Germania.}

Durch Weiterführung der Berechnung der durch Jupiter und Saturn bewirkten Störungen gelangte ich zu folgenden Elementen des Planeten (241) Germania.

Epoche 1893 April $300^{\text {h }}$ M. Z. Berlin

\begin{tabular}{|c|c|c|c|c|c|c|c|c|}
\hline \multicolumn{2}{|c|}{1893} & \multicolumn{2}{|c|}{$\alpha$ app. } & \multicolumn{3}{|c|}{$\delta$ app. } & $\log \Delta$ & Ab.-Z. \\
\hline pril & 3 & $13^{h} 53^{-}$ & $3^{5.84}$ & $-19^{\circ}$ & $1 I^{\circ}$ & $54{ }^{n} \mathrm{I}$ & 0.359367 & $19^{\mathrm{m}} 1^{\mathrm{s}}$ \\
\hline & 4 & $5^{2}$ & 21.50 & 19 & 8 & 29.9 & $0.35^{8} 345$ & $185^{8}$ \\
\hline & 5 & $5 \mathrm{I}$ & 38.54 & 19 & 4 & 58.5 & 0.357373 & 56 \\
\hline & 6 & 50 & 55.03 & 19 & I & 20.0 & 0.3 & 54 \\
\hline & 7 & 50 & 10.98 & 18 & 57 & $34 \cdot 7$ & 0.3 & $5^{I}$ \\
\hline & 8 & 49 & 26.45 & 18 & 53 & $42 \cdot 5$ & 0.3 & 49 \\
\hline & 9 & 48 & 41.49 & I 8 & 49 & 43.8 & 0.3 & 47 \\
\hline & to & 47 & & I 8 & 45 & $3^{8.7}$ & $\circ 3$ & 45 \\
\hline & I I & 47 & 10.43 & I 8 & 41 & $27 \cdot 4$ & 0.3 & 44 \\
\hline & I 2 & 46 & 24.42 & I 8 & 37 & 10.0 & 0.3 & 42 \\
\hline & 13 & 45 & 38.16 & I 8 & 32 & 46.9 & 464 & 41 \\
\hline & 14 & 44 & 51.70 & I 8 & 28 & 18.2 & 969 & 39 \\
\hline & 15 & 44 & 5.08 & I 8 & 23 & 44.2 & 529 & $3^{8}$ \\
\hline & 16 & 43 & 18.36 & I 8 & 19 & $5 \cdot 2$ & 146 & 37 \\
\hline & 17 & 42 & 31.59 & I 8 & 14 & 21.4 & 0.349818 & 36 \\
\hline 8 & 18 & 41 & & I 8 & 9 & 33.2 & 547 & $3^{6}$ \\
\hline & 19 & 40 & 58.09 & 18 & 4 & 40.7 & 333 & 35 \\
\hline & 20 & 40 & I 1.46 & I 7 & 59 & $44 \cdot 4$ & 175 & 35 \\
\hline & 2 I & 39 & 24.97 & 17 & 54 & $44 \cdot 4$ & 0.349073 & 34 \\
\hline & 22 & 38 & $3^{8.69}$ & 17 & 49 & 41.2 & 0.34 & 34 \\
\hline & 23 & 37 & 52.64 & I 7 & 44 & 34.9 & 0.349039 & 34 \\
\hline & 24 & 37 & 6.89 & 17 & 39 & 25.9 & 0.349106 & 1835 \\
\hline
\end{tabular}

$$
\begin{aligned}
& M=238^{\circ} \text { I } 8^{\prime} \text { I } 4^{\prime \prime} 9 \\
& \pi=342 \text { II } 49.5 \\
& \Omega=2722145.9 \\
& \varphi=5^{\circ} 35^{\circ} 37^{\prime \prime} 7 \\
& \mu=665^{\prime \prime} 5055^{\circ} \\
& i=53046.2 \\
& \log a=0.4845699
\end{aligned}
$$

\begin{tabular}{|c|c|c|c|c|c|c|c|c|}
\hline \multicolumn{2}{|c|}{1893} & \multicolumn{2}{|c|}{$\alpha$ app. } & \multicolumn{3}{|c|}{ d app. } & $\log \Delta$ & Ab.-Z. \\
\hline Apri & 24 & $13^{\mathrm{h}} 37$ & m $\quad 6.89$ & $-I \eta^{\circ}$ & $39^{\prime}$ & $25: 9$ & 0.349106 & $18^{m} 35^{s}$ \\
\hline & 25 & $3^{6}$ & 21.46 & 17 & 34 & 14.6 & 0.349229 & 35 \\
\hline & 26 & 35 & 36.41 & I 7 & 29 & I.I & 0.349407 & 35 \\
\hline & 27 & 34 & 51.78 & 17 & 23 & 45.8 & $0.34964 I$ & 36 \\
\hline & 28 & 34 & 7.61 & 17 & 18 & 29.0 & 0.349928 & 37 \\
\hline & 29 & 33 & 23.94 & 17 & I 3 & I I.O & 0.350270 & $3^{8}$ \\
\hline & 30 & $3^{2}$ & 40.82 & 17 & 7 & 52.0 & 0.350666 & 39 \\
\hline Mai & $\mathbf{I}$ & $3 I$ & 58.27 & 17 & 2 & $32 \cdot 5$ & $0.3511 \times 6$ & 40 \\
\hline & 2 & 31 & 16.34 & I 6 & 57 & I 2.6 & 0.351617 & $4 I$ \\
\hline & 3 & 30 & 35.07 & 16 & 51 & 52.7 & 0.352171 & 42 \\
\hline & 4 & 29 & 54.49 & 16 & 46 & 33.1 & 0.352777 & 44 \\
\hline & 5 & 29 & 14.64 & I 6 & $4 I$ & 14.1 & 0.353433 & 46 \\
\hline & 6 & 28 & 35.55 & I 6 & 35 & 55.9 & 0.354140 & 48 \\
\hline & 7 & 27 & 57.27 & I 6 & 30 & 38.9 & 0.354896 & 49 \\
\hline & 8 & 27 & $19.8 \mathrm{I}$ & 16 & 25 & 23.4 & 0.355701 & 52 \\
\hline & 9 & 26 & $43.2 I$ & 16 & 20 & $9 \cdot 7$ & $0.35^{6555}$ & 54 \\
\hline & IO & 26 & $7 \cdot 5 \mathrm{I}$ & 16 & 14 & 57.9 & 0.357455 & $5^{6}$ \\
\hline & I I & 25 & 32.73 & 16 & 9 & 48.5 & $0.35^{8402}$ & 1859 \\
\hline & 12 & 24 & $58.9 \mathrm{I}$ & I 6 & 4 & $4 \pi \cdot 7$ & 0.359394 & 191 \\
\hline & 13 & 24 & 26.06 & I 5 & 59 & 37.8 & $0.3^{60431}$ & 4 \\
\hline & I 4 & 23 & $54.2 \cdot 3$ & I 5 & 54 & 37.1 & $0.36 \times 512$ & 7 \\
\hline & 15 & 1323 & 23.44 & 15 & 49 & 39.9 & 0.362635 & 1910 \\
\hline
\end{tabular}

Ephemeride für I $^{\mathrm{h}}$ M. Z. Berlin.

Grösse 19 m 5 .

Düsseldorf 1893 Febr. 24. 